\title{
Changement, incertitude et gestion en éducation
} Regards sur la réforme scolaire au Québec

\section{Change, Uncertainty and Management in Education} A Look at Educational Reform in The Province of Quebec

\section{Cambio, incertidumbre y gestión en educación Reflexiones sobre la reforma escolar en Quebec}

\section{Jean J. Moisset et Alice Couture}

Volume 29, numéro 2, automne 2001

Origines et incidences des nouveaux rapports de force dans la gestion de l'éducation

URI : https://id.erudit.org/iderudit/1079556ar

DOI : https://doi.org/10.7202/1079556ar

Aller au sommaire du numéro

Éditeur(s)

Association canadienne d'éducation de langue française

ISSN

0849-1089 (imprimé)

1916-8659 (numérique)

Découvrir la revue

Citer cet article

Moisset, J. \& Couture, A. (2001). Changement, incertitude et gestion en

éducation : regards sur la réforme scolaire au Québec. Éducation et

francophonie, 29(2), 169-197. https://doi.org/10.7202/1079556ar

\section{Résumé de l'article}

Phénomène marquant dans l'évolution des systèmes éducatifs de la plupart des pays du monde, les réformes sont à la fois porteuses de changement et d'incertitude, avec d'importantes implications pour la gestion en éducation. Cela est encore plus vrai dans le contexte de sociétés industrielles avancées, où à l'accélération du changement s'ajoute la complexité des organisations, due, au moins en partie, à l'éclatement des systèmes traditionnels de valeurs. Sur la base de cette problématique, cet article procède d'une réflexion et d'une recherche exploratoire. Il est articulé autour de trois axes : 1 . Le premier concerne le concept d'incertitude dont nous présentons, au-delà de sa définition, les principaux facteurs qui y sont associés ainsi que leur influence sur la gestion, en milieu scolaire notamment; 2. Le deuxième examine, sur un plan général, certaines stratégies devant permettre de gérer de manière plus ou moins efficace en situation d'incertitude, cette analyse débouchant sur la gestion participative et les conditions de succès d'application de ce modèle; 3 . Le troisième axe, à partir du substrat théorique dégagé de ce qui précède, analyse les données recueillies relativement aux perceptions/opinions d'un échantillon de douze directeurs et directrices d'école de la région de Québec quant aux changements en cours et envisagés dans le cadre de l'actuelle réforme scolaire du Québec, notamment en ce qui concerne la décentralisation de certains pouvoirs vers les établissements scolaires. Il se dégage que le modèle de gestion participative, moyennant certaines conditions, est susceptible de mobiliser les acteurs de l'école, malgré un certain climat d'incertitudes et de tensions inévitables liées aux changements en cours.

Tous droits réservés ( $\odot$ Association canadienne d'éducation de langue française, Ce document est protégé par la loi sur le droit d’auteur. L’utilisation des 2001 services d'Érudit (y compris la reproduction) est assujettie à sa politique d'utilisation que vous pouvez consulter en ligne.

https://apropos.erudit.org/fr/usagers/politique-dutilisation/ 


\section{Changement, incertitude et gestion en éducation Regards sur la réforme scolaire au Québec}

Jean J. MOISSET, professeur

Faculté des sciences de l'éducation, Université Laval (Québec), Canada

Alice COUTURE, professeure

Faculté des sciences de l'éducation, Université Laval (Québec), Canada

\section{RÉSUMÉ}

Phénomène marquant dans l'évolution des systèmes éducatifs de la plupart des pays du monde, les réformes sont à la fois porteuses de changement et d'incertitude, avec d'importantes implications pour la gestion en éducation. Cela est encore plus vrai dans le contexte de sociétés industrielles avancées, où à l'accélération du changement s'ajoute la complexité des organisations, due, au moins en partie, à l'éclatement des systèmes traditionnels de valeurs. Sur la base de cette problématique, cet article procède d'une réflexion et d'une recherche exploratoire. Il est articulé autour de trois axes:

1. Le premier concerne le concept d'incertitude dont nous présentons, au-delà de sa définition, les principaux facteurs qui y sont associés ainsi que leur influence sur la gestion, en milieu scolaire notamment;

2. Le deuxième examine, sur un plan général, certaines stratégies devant permettre de gérer de manière plus ou moins efficace en situation d'incertitude, cette analyse débouchant sur la gestion participative et les conditions de succès d'application de ce modèle; 
3. Le troisième axe, à partir du substrat théorique dégagé de ce qui précède, analyse les données recueillies relativement aux perceptions/opinions d'un échantillon de douze directeurs et directrices d'école de la région de Québec quant aux changements en cours et envisagés dans le cadre de l'actuelle réforme scolaire du Québec, notamment en ce qui concerne la décentralisation de certains pouvoirs vers les établissements scolaires.

Il se dégage que le modèle de gestion participative, moyennant certaines conditions, est susceptible de mobiliser les acteurs de l'école, malgré un certain climat d'incertitudes et de tensions inévitables liées aux changements en cours.

\section{ABSTRACT}

\section{Change, Uncertainty and Management in Education : A Look at Educational Reform in The Province of Quebec}

Jean J. MOISSET, professeur

Faculté des sciences de l'éducation, Université Laval (Québec), Canada

Alice COUTURE, professeure

Faculté des sciences de l'éducation, Université Laval (Québec), Canada

A determining phenomenon in the evolution of education systems in most countries in the world, reforms bring both change and uncertainty, with important implications for management in education. This is even truer in the context of advanced industrial societies, where the complexity of organizations, due, at least in part, to the breakdown of the traditional value system, is added to the acceleration of change. On the basis of this problem, this article proceeds with an exploratory reflection and research. It is based on three main ideas:

1. The first deals with the concept of uncertainty, of which we present, beyond its definition, the main factors associated with it, as well as their influence on management, particularly in the school setting.

2. The second examines, on a general level, certain forward-looking strategies allowing a management style that is more or less effective in a situation of uncertainty. This analysis explores participatory management and the conditions for success in the application of this model.

3. The third idea, based on the theoretical substratum of the preceding, analyzes the data collected related to the perceptions/opinions of a sample of twelve school principals from the region of Quebec, about the planned changes and those now taking place in the framework of Quebec's current education reform, particularly concerning the decentralization of certain powers towards the schools.

It appears that, under certain conditions, the model of participatory management may mobilize those working in the school, despite an inevitable climate of uncertainty and tension linked to the changes in progress. 


\section{RESUMEN}

\section{Cambio, incertidumbre y gestión en educación : Reflexiones sobre la reforma escolar en Quebec}

Jean J. MOISSET, professeur

Faculté des sciences de l'éducation, Université Laval (Québec), Canada

Alice COUTURE, professeure

Faculté des sciences de l'éducation, Université Laval (Québec), Canada

Fenómeno notable en la evolución de los sistemas educativos de la mayor parte de los países del mundo, las reformas son a la vez portadoras de cambios y de incertidumbre, con implicaciones importantes para la gestión en educación. Esto es aún más cierto en el contexto de las sociedades industriales avanzadas, en donde a la aceleración del cambio se agrega la complejidad de las organizaciones debida, al menos en parte, al estallido de los sistemas tradicionales de valores. A patir de esta problemática, este artículo es el producto de una reflexión y de una investigación exploratoria. Se articula al rededor de tres ejes :

1. El primero aborda el concepto de incertidumbre del cual presentamos, más que su definición, los factores principales que se le asocian así como su influencia sobre la gestión, especialement en el medio escolar;

2. El segundo examina, de manera general, ciertas estrategias que permiten gestionar de manera más o menos eficaz en situación de incertidumbre, análisis que conduce hacia la gestión participativa y las condiciones óptimas para la aplicación de este modelo;

3. El tercer eje, a partir de un substrato teórico proveniente de lo que precede, analiza los datos recogidos sobre las percepciones/opiniones de una muestra de doce directores y directoras de escuela de la región de Quebec sobre los cambios que se están viviendo, considerados en el cuadro de la actual reforma escolar de Quebec, principalmente en lo que concierne a la decentralización de ciertos poderes hacia los establecimientos escolares.

Se desprende que el modelo de gestión participativa, si se consideran ciertas condiciones, es capaz de mobilizar a los actores de la escuela, a pesar de un cierto clima de incertidumbre y de las tensiones inevitables ligadas a los cambios en curso.

\section{Introduction}

Le secteur de l'éducation, en Amérique du Nord comme ailleurs, a été le théâtre d'une multitude de projets de réforme, de manière générale mais à des degrés variables, porteurs de changement, d'incertitudes et souvent de stress. À en croire 
plusieurs chercheurs, entre autres House (1996) et Pai et Adler (1997), si l'on se réfère à l'expérience passée, ces réformes et les théories qui les sous-tendent ont eu très peu d'impact sur les pratiques à l'école, sur la manière dont les écoles sont gérées et les enfants éduqués. C'est que souvent les initiateurs de ces réformes sont des « outsiders " plutôt que des intervenants confrontés dans le quotidien aux problèmes des milieux concernés.

Dans la mouvance d'une tradition de réforme déjà vieille de plus de trois décennies et suite aux derniers États généraux sur l'éducation et à l'adoption au Québec en 1997 de la loi 180, de multiples changements sont projetés ou déjà en cours dans le milieu scolaire, communément catalogués sous la dénomination de réforme Marois, du nom de la ministre de l'Éducation d'alors.

Globalement, les mesures envisagées par l'actuelle réforme scolaire au Québec (loi 180) visent un nouveau partage des pouvoirs entre les commissions et les établissements scolaires, devant déboucher sur un rapprochement des pouvoirs de décision et de gestion du niveau de la base, c'est-à-dire de l'école elle-même, désormais "placée sous la responsabilité partagée de sa direction et d'un conseil d'établissement ». Cette réforme devrait favoriser une plus large participation des acteurs de l'école aux décisions touchant son fonctionnement, avec comme résultat, espèret-on, une amélioration de la vie à l'école et de la réussite scolaire des élèves. Il n'en reste pas moins, à entendre certains propos dans les milieux concernés, que les changements envisagés sont porteurs de beaucoup d'incertitudes, ce qui entraîne, du même coup, un certain scepticisme: incertitude quant au degré de préparation des bénéficiaires du nouveau partage des pouvoirs, incertitude quant aux réactions, dans la pratique, des anciens détenteurs de ces pouvoirs, etc. Quoi qu'il en soit, les perceptions et le degré d'engagement des acteurs concernés constituent, presque à coup sûr, une condition majeure du succès de l'implantation de cette réforme. D'où la pertinence, voire l'importance de connaître les points de vue de ces acteurs, et notamment des directeurs et directrices d'école, par rapport à ces changements. À partir d'un schéma d'entretien, nous avons voulu savoir ce que pensent un échantillon de directeurs et directrices d'école de la région de Québec par rapport à la réforme scolaire et, en particulier, comment ils perçoivent les mesures envisagées quant à leur impact sur leur charge de travail et les modes d'exercice de leurs fonctions. Cet article, prenant appui sur la littérature relative à la problématique du changement en éducation et nos réflexions sur ce sujet, présente et analyse les principaux résultats de cette étude tout à fait exploratoire.

De manière plus spécifique, nous soutenons ici la thèse qu'aujourd'hui la gestion scolaire est de loin plus complexe qu'hier, l'incertitude et la vitesse du changement étant beaucoup plus élevées pour plusieurs raisons, dont nous essaierons de mettre en relief celles qui paraissent les plus importantes. Nous partons également du postulat que le défi de la gestion en période de réforme peut être relevé. Deux hypothèses sous-tendent ce postulat, à savoir, d'une part, qu'il est possible de réduire la marge d'incertitude inhérente à la vie de toute organisation et, d'autre part, qu'il est possible pour les gestionnaires et les membres des organisations d'avoir une commune définition du FUTUR de leur organisation. 
Ce texte sera articulé autour de trois axes :

1. le premier concerne le concept d'incertitude dont nous présenterons, au-delà de sa définition, les facteurs majeurs qui y sont associés ainsi que leur influence sur la gestion, en milieu scolaire notamment;

2. le deuxième examinera, sur un plan général, certaines stratégies devant permettre de gérer de manière plus ou moins efficace en situation d'incertitude, cette analyse débouchant sur la gestion participative et les conditions de succès d'application de ce modèle;

3. le troisième axe, à partir du substrat théorique dégagé de ce qui précède, analysera les données recueillies relativement aux opinions/perceptions des directeurs d'école quant aux changements envisagés et en cours dans le cadre de la réforme scolaire du Québec, notamment en ce qui concerne la décentralisation des pouvoirs vers les établissements scolaires.

\section{Incertitude, changement et gestion en éducation}

«Il est peu douteux que le thème de l'incertitude soit récurrent dans l'imaginaire humain", déclare Bronner (1997, p. 7) et de son côté, rejoignant en quelque sorte le philosophe, l'expert en gestion financière souligne que «l'incertitude a toujours été consubstantielle au travail managérial, au quotidien comme en matière de stratégies" (Boury, 1996, p. 175). Dans ce qui semble être un concert, le spécialiste de l'éducation n'est pas en reste et va même plus loin, mettant en relief le fait que "l'éducation est un métier impossible, parce que c'est un métier complexe, qui oblige à affronter des contradictions irréductibles, tant dans l'esprit de l'acteur que dans les rapports sociaux " (Perrenoud, 1996, p. 17). Il ne fait pas de doute que la gestion scolaire est, aujourd'hui plus que jamais, marquée par l'incertitude et la complexité. À cet égard, Perrenoud toujours soutient que «l'école est moins que jamais sûre de son action et qu'aucune réforme scolaire ne peut aujourd'hui garantir qu'elle apportera un progrès décisif " (1996, p. 30). Nous examinerons dans les sections qui suivent les notions d'incertitude et de complexité, de même que les principaux facteurs qui y sont associés.

\section{Incertitude et complexité : les notions}

Le terme «incertitude» couramment utilisé dans la vie de tous les jours ne semble pas poser de problème pour sa définition. Il n'en reste pas moins pertinent d'éclaircir en partant cette notion. L'incertitude est liée indissolublement au nonsavoir et au futur et, de manière quasi tautologique, on peut dire que c'est l'inconnu qui est à la source de l'incertitude, qui est l'incertitude. En fait, le réel, dans quelque secteur où il se trouve, sous quelque angle on l'examine, même au temps présent, ne se laisse jamais tout à fait cerner. Cela est encore plus vrai si ces réalités, faits de nature ou de culture, sont regardées dans la dynamique de leur évolution. Les lois de la causalité ou un certain déterminisme des choses peuvent nourrir l'illusion d'une connaissance et d'une maîtrise des faits de nature (et encore!) traduites par le progrès 
phénoménal de la science et de la technique, enregistré de la révolution industrielle à nos jours. Mais force est de reconnaître que les faits de culture, avec la marge de liberté individuelle de l'acteur humain et l'imprévisibilité du futur, ne sont jamais complètement circonscrits et encore moins maîtrisés.

Nous ne sommes pas loin de la définition que donne Bronner de l'incertitude, «terme», souligne-t-il $(1997$, p. 3), fréquemment utilisé dans deux sens qui ne se recoupent pas exactement. On peut tout d'abord être incertain relativement à un événement futur sur lequel nous n'avons pas tout pouvoir : on parlera alors d'incertitude en finalité. On peut par ailleurs être en état d'incertitude concernant des objets bien différents - par exemple, est-il bien ou mal d'accepter l'avortement? Nous proposons pour ces cas de parler d'incertitude de sens.» La deuxième forme d'incertitude n'est pas loin, à notre point de vue, du concept de « complexité » qu'Edgar Morin (1977, p. 379) définit comme «l'association de ce qui était considéré comme antagoniste [et qui] correspond, dans ce sens, à l'irruption des antagonismes au cœur des phénomènes organisés".

On peut faire remarquer ici qu'au-delà de cette distinction tout à fait pertinente, les deux formes d'incertitude, en finalité ou de sens, sont sous-tendues et s'expliquent par le non-savoir associé, dans le premier cas, à un futur non prévisible et, dans le second, à des échelles de valeurs divergentes. Nous savons toute l'importance qu'ont les valeurs dans le système d'éducation et dans le processus de planification et de gestion de l'éducation. Ainsi, souligne Burns (1995, p. 79), « the major emphasis is on conceptual techniques for prioritizing and operationalizing values, that is, on clarifying values choices and bringing values and actions (ends and means) into harmony». André Godin (2000) fait valoir de son côté que "dans tous les modèles et expériences du projet éducatif que nous avons analysés, il y avait un point commun, la présence des valeurs. Tout le monde est d'avis que c'est un élément incontournable dans la conception d'un projet éducatif. " Les valeurs sont en quelque sorte à la base de l'éducation et du changement en éducation. Encore faut-il savoir les rendre convergentes et s'entendre sur la place qu'on veut leur accorder! C'est là un défi important auquel sont confrontés, aujourd'hui plus que jamais, les gestionnaires d'établissement scolaire.

En réalité, contrairement à ce qu'on a pu croire ou laisser croire, l'incertitude et la complexité ne sont pas des phénomènes nouveaux, dans la mesure où elles peuvent être considérées comme des caractéristiques inhérentes à tout ce qui existe et notamment à tous les systèmes vivants, qu'il s'agisse d'individus, de groupes, d'organisations ou de sociétés. Mais ce n'est pas un effet du hasard si, depuis le début des années 1970, une attention soutenue et quasi permanente a été accordée à ces phénomènes par les chercheurs, en quête d'un schéma de théorisation ou de modélisation d'une situation que d'aucuns n'hésitent pas à qualifier de turbulence ou de chaos (Prigogine, 1990; Passis-Pasternak, 1991; Bardot, 1996; Bourgeault, 1985). Il nous paraît indiqué de nous arrêter brièvement à quelques manifestations de la « crise » liée à l'incertitude et à la complexité, notamment dans le monde scolaire, et aux principaux facteurs qui y sont associés. 


\section{Effets et causes}

À l'instar du phénomène de turbulence qui évoque l'agitation et le désordre, la quasi-impossibilité d'une prévision sérieuse, l'incertitude et la complexité découlent d'une série d'antagonismes, dont Perrenoud (1996, p. 25) rappelle plusieurs exemples pour le domaine de l'éducation: "contradictions entre la personne et la société, l'unité et la diversité, la dépendance et l'autonomie, l'invariance et le changement, l'ouverture et la fermeture, l'harmonie et le conflit, l'égalité et la différence. Chacune de ces contradictions, poursuit l'auteur, opère à divers niveaux de l'organisation scolaire, celui de la salle de classe... celui de l'établissement, celui du système éducatif dans son ensemble."

Il y aurait lieu d'analyser chacune de ces dyades et d'examiner comment, concrètement, elles influent sur le fonctionnement du système scolaire à ses divers niveaux. Pour faire bref, nous dirons que toutes ces contradictions qui traversent l'organisation scolaire sont étroitement liées à l'éclatement de notre système de valeurs traditionnelles, qui a entraîné la privation momentanée des points de repère et d'ancrage sur lesquels éducateurs et gestionnaires de l'éducation pouvaient s'appuyer pour guider leur action en matière d'enseignement et de gestion. La remise en question des règles du jeu et des normes de fonctionnement des organisations scolaires, associées au principe du respect de l'autorité sous-jacent au modèle bureaucratique et hiérarchisé, si légitime soit-elle, n’a pas moins créé un vide. De toute manière, tout en reconnaissant les pas importants que ce modèle a permis à l'éducation de faire au cours des dernières décennies, le Conseil supérieur de l'éducation (1993) ne plaidait pas moins pour un autre modèle de gestion, "un modèle de référence, s'inspirant davantage d'une logique conviviale... axé sur une vision des choses à plus long terme, sur le sens de la mission éducative et sur le souci de planification stratégique des activités " (CSE, 1995, p. 16). Ce sont là précisément des défis qu'incertitude et complexité rendent sinon impossibles du moins difficiles à relever. De toute façon, une question demeure: quelle est la marge de jeu du gestionnaire pour ne pas recréer un autre modèle bureaucratique en se donnant de nouveaux points de repère et d'ancrage? La préoccupation associée à cette question sera présentée dans les sections qui vont suivre.

\section{Stratégies en situation d'incertitude et de complexité}

\section{Postulat et hypothèse}

On peut partir du postulat que, de manière générale, par une propension naturelle, l'être humain a besoin de cohérence et de sens pour son existence, comme individu pris isolément ou comme membre d'un groupe, d'une organisation ou d'une société. Il est ainsi rassurant de pouvoir énoncer comme hypothèse plausible que toute réduction d'incertitude, de sens ou de finalité sera considérée comme un objectif valable aux yeux des membres d'une organisation scolaire par exemple. On ne restera pas moins confronté au problème de la démarche à emprunter en vue de la définition et de la mise en œuvre des stratégies appropriées. Parler du problème de 
l'incertitude et de la complexité ou de stratégies, face à l'incertitude et à la complexité, n'a donc de sens qu'en considération d'individus, de personnes capables de décisions et d'action devant une situation donnée.

\section{Une typologie des situations d'incertitude}

Les stratégies à développer dépendent dans une certaine mesure des caractéristiques de la situation d'incertitude, dont Bronner (1997, p. 57) distingue trois types, au moins pour l'incertitude en finalité.

Dans un premier type, la marge de manœuvre de l'acteur est la plus étroite, soit parce que ce dernier n'y a aucun intérêt, soit parce que la situation est complètement en dehors de son pouvoir. Cette situation se traduit bien par les propos qui nous sont à tous plus ou moins familiers, du genre : je n'y peux rien ou je ne veux rien en savoir.

Le deuxième type de situation d'incertitude offre une marge de manœuvre plus importante à l'acteur qui a le choix entre accepter l'incertitude et en payer éventuellement les conséquences ou l'éviter, ce qui comportera un coût plus ou moins certain selon le cas. Cette incertitude découle d'un événement qui vient rompre le quotidien de l'acteur, c'est-à-dire la représentation qu'il se fait du monde relativement stable dans lequel il vit, auquel il s'est adapté et par rapport auquel il établit ses anticipations.

Un exemple de la situation II peut être trouvé dans les programmes de retraite anticipée (ou de départ volontaire) qui ont été mis sur pied, mais assortis dans certains cas d'une opération d'évaluation pour tous les employés de l'organisation, âgés de 55 ans et plus.

Les alternatives sont les suivantes:

- On se retire volontairement et une prime est offerte, équivalant à une fraction du salaire annuel, déterminée par le nombre d'années de service qu'on pourrait encore donner, variant entre 1 et 10. Décision à prendre dans un délai de 9 mois. - Dans un délai de 15 mois, il est prévu que tous les employés concernés seraient systématiquement évalués et que, selon les résultats, certains pourraient être congédiés, évidemment sans prime aucune.

Jusqu'au surgissement de ces programmes, les employés évoluaient suivant leurs représentations, dans un univers stable où ils anticipaient de prendre leur retraite en fonction de leurs besoins et, possiblement, jusqu'à l'âge de 65 ans. L'incertitude ainsi créée ne dépend pas de l'employé, mais celui-ci a le choix entre l'accepter, c'est-à-dire décider de ne pas prendre la retraite anticipée, acceptant du même coup de se faire évaluer le moment venu et de se faire éventuellement congédier avec les conséquences négatives que cela comporterait, ou éviter cette incertitude en acceptant de prendre sa retraite, renonçant en même temps et de manière plus ou moins certaine aux avantages qu'il aurait pu retirer en travaillant jusqu'à 65 ans.

Cet exemple n'est pas seulement théorique, puisque c'est la situation qui a été vécue depuis le début des années 1990 par les employés (cadres techniques et administratifs) de la fonction publique au Québec et ailleurs au Canada et par les professeurs de la plupart des universités au pays. 
Dans la troisième situation typique d'incertitude, non seulement l'acteur peut éviter cette incertitude, mais encore c'est lui-même qui la génère. Bronner (1997, p. 74), citant Menger (1991), donne l'exemple de «l'apprenti artiste», «quand il décide de se lancer dans la voie très incertaine (socio-économiquement parlant) de la production artistique». Mais là, les anticipations des gains possibles (devenir millionnaire) sont très élevées de même que les satisfactions psychologiques (vedettariat international).

Somme toute, il serait vain de penser à la possibilité d'établir une série de stratégies entre lesquelles il n'y aurait qu'à choisir selon les situations d'incertitude infiniment nombreuses face auxquelles on peut se trouver. Il faudrait en outre compter avec la variabilité également très élevée des appréciations de ces situations selon les acteurs et les contextes. Cela dit, deux éléments majeurs peuvent être dégagés qui nous semblent transversaux par rapport aux situations typiques d'incertitude décrites précédemment, à savoir l'information et l'autonomie de l'acteur. Ces deux éléments sont au cœur non pas d'une stratégie de l'incertitude et de la complexité, mais d'une démarche pour le développement de stratégies face à l'incertitude et à la complexité particulièrement dans le domaine de la gestion éducative. C'est ce que nous allons voir, après avoir évoqué, à grands traits, des stratégies historiquement développées, soit pour amadouer, soit pour éviter l'incertitude et la complexité.

\section{Démarches en situation d'incertitude et de complexité}

De tout temps, comme il a été souligné précédemment, l'être humain a vécu en situation d'incertitude et a cherché des moyens de réduire l'incertitude. Remontant dans la nuit des temps, l'on retrouve les mythes et les superstitions qui semblent avoir survécu au progrès des sciences et de la technique, quand on voit en cette fin de millénaire la montée des intégrismes, des fondamentalismes et la multiplication des sectes et OTS (Organisation du Temple solaire) de tout acabit. Ces «stratégies" semblent se développer d'autant plus que «l'incertitude est désormais un état et non plus une donnée», pour reprendre le mot de Patrick Boury (1996, p. 176). On ne saurait du reste les rejeter du revers de la main, car pour avoir la vie si dure, c'est qu'ils répondent à un besoin. Il faut reconnaître toutefois que, sécurisantes, et même euphorisantes pour les individus, ces stratégies ne laissent pas moins les problèmes intacts, quand ils ne deviennent pas plus graves au niveau des organisations.

Un deuxième groupe de stratégies existe qui consiste à éviter ou à nier l'incertitude, telle qu'elle se manifeste au temps présent. Dans un premier cas, les acteurs se détournent en un sens des problèmes du moment pour se réfugier dans un passé, jugé ou perçu comme plus confortable. C'est la nostalgie du bon vieux temps. Mais comme on sait, un retour pur et simple aux valeurs ou aux pratiques anciennes s'avère le plus souvent inutile sinon impossible. Dans un deuxième cas, les acteurs pratiqueront la stratégie de l'enfermement ou du repliement sur eux-mêmes, en marge des incertitudes et de l'insécurité de la société globale. Cette deuxième stratégie n'est pas sans lien avec les mythes dont il a été question plus haut et avec la troisième stratégie de l'évitement des incertitudes du présent par la fuite vers le futur (millénarisme, fin des temps). Mais là encore, si des individus et des groupes y 
trouvent leur compte, ce n'est pas le cas pour les problèmes collectifs qui restent entiers avec leur incertitude et leur complexité. Peut-on sortir de ce qui semble être un cul-de-sac? La démarche proposée ci-après apporte au moins quelques éléments de réponse à la question.

Elle s'articule autour de trois pôles opérationnels en interaction synergique, procédant à la fois de la logique sous-jacente au processus rationnel de résolution des problèmes dans leur séquence et leur itérativité, de la pensée systémique dans la saisie des liens que les problèmes ont entre eux et avec leur environnement et d'une philosophie de l'action, centrée sur la reconnaissance et la mobilisation de tous les acteurs de l'organisation. À la vérité, il n'y a là rien de nouveau, mais ne dit-on pas qu'il n'y a rien de mieux que les vieilles casseroles pour concocter et mijoter de bons petits plats... C'est ce à quoi sans doute a pensé Perrenoud quand il a défini (1996, p. 40) "quelques lignes de conduite face à la complexité » :

1) la reconnaître et ne pas faire comme si on la dominait tout seul;

2) l'analyser et l'apprivoiser ensemble;

3) mettre en place des institutions systémiques.

La première étape, dans tout processus de résolution de problème, c'est la reconnaissance ou l'identification du problème. Gérer l'incertitude et la complexité passe d'abord par une reconnaissance de cette complexité et de cette incertitude, dont, en éducation comme dans d'autres secteurs, le changement et la contradiction constituent des facteurs clés. Il importe non seulement de reconnaître mais d'accepter que l'incertitude et la complexité sont des caractéristiques des fonctions de l'éducateur et du gestionnaire de l'éducation, fonctions que ni l'un ni l'autre ne saurait accomplir efficacement seul. De fait, gérer l'incertitude et la complexité, en éducation comme ailleurs, ne peut être l'affaire d'un seul gestionnaire, mais de l'ensemble des acteurs concernés par le fonctionnement de l'organisation scolaire, enseignants, élèves, parents d'élèves, représentants du milieu, et par la réalisation de la mission éducative.

L'on admettra toutefois que cet "âge de la majorité », désormais reconnu à l'ensemble des acteurs plutôt qu'aux seules têtes dirigeantes, est susceptible de créer de nouveaux rapports de force, voire des contradictions sur la scène scolaire. Cela est même inévitable, compte tenu de la pluralité des perceptions et des visions des uns et des autres par rapport aux problèmes auxquels l'école est confrontée. C'est là qu'un certain type de leadership en matière de gestion peut s'avérer capital pour rendre convergents les divers points de vue et transformer en tensions créatrices ce qui était au départ perçu comme des contradictions.

Cette manière de voir et de faire va au-delà d'une logique classique dont le souci, au plan de l'organisation et de la gestion, a été une définition claire des rôles et des responsabilités de chacun correspondant à un schéma de division du travail garant d'une plus grande efficacité. C'est tout le monde et pas seulement le directeur ou la directrice de telle organisation qui est responsable de la reconnaissance de la complexité, reconnaissance du reste nécessaire mais non suffisante pour bien la gérer. 
Nous retombons ici dans la deuxième étape du processus de résolution de problème, qui est son analyse. Analyser la complexité, au-delà de l'identification de ses manifestations, c'est la saisir dans ses tenants (causes) et ses aboutissants (effets), cela de manière propre au système éducatif, à l'organisation ou à l'établissement scolaire concernés. Une fois encore, sans méconnaître les mérites ou les apports de la spécialisation et de la professionnalisation, c'est l'ensemble des acteurs qui sont le plus aptes à analyser, dans leur incertitude et leur complexité, les problèmes auxquels eux et leur organisation sont confrontés et les possibles solutions à envisager.

À ce dernier égard, il semble que l'on n'a pas dépassé la bonne vieille analyse de système qui, soulignait Barel (1971, p. 72), «consiste, dans la plupart des cas, à mettre en valeur le fait que l'objectif examiné doit être replacé dans le contexte plus large que son système originel, le terme d'analyse servant à souligner qu'il est utile de décomposer les problèmes complexes en éléments constituants». Réexprimant ces idées devenues classiques, nous pouvons dire que la pensée systémique accorde une importance fondamentale à considérer les situations et les réalités dans leur configuration globale et à les resituer dans leur contexte et leur milieu. Elle accorde, en outre, une attention spéciale aux relations et interactions entre les éléments au sein et constitutifs des systèmes et à leurs rapports avec l'environnement. C'est à peu près dans les même termes qu'à la suite d'Edgar Morin parle Perrenoud (1996, p. 43) face à la complexité, « une institution doit être capable de pensée systémique, c'està-dire, capable de se penser dans sa complexité interne et ses dépendances externes, de construire une vue d'ensemble de son fonctionnement et de son environnement, et de proposer des lignes d'action cohérentes».

Somme toute, ce qui se dégage des développements qui précèdent, c'est qu'une gestion efficace de l'incertitude et de la complexité, plus que par leur négation, leur réduction et encore moins leur évitement, est conditionnée par la mise en place des voies et moyens d'une commune appréciation de l'incertitude et de la complexité par l'ensemble des membres de l'organisation. Comme le souligne Bronner (1997, p. 124), «il est en effet peu probable, et pas nécessairement souhaitable, que l'incertitude soit un jour parfaitement circonscrite». Gérer la complexité et l'incertitude avec une probabilité raisonnable de succès consistera de plus en plus, pour les décideurs politiques et les administrateurs de l'éducation, à mettre tout le monde dans le coup, à partager les problèmes avec l'ensemble des acteurs et en particulier ceux qui sont les plus directement concernés. Qu'en a-t-il été au niveau de la démarche qui a abouti à la réforme scolaire, appelée au moins au début réforme Marois, du nom de la ministre de l'Éducation d'alors? Qu'en disent les directeurs et directrices d'établissements scolaires si impliqués déjà dans sa mise en œuvre? 


\section{La réforme scolaire : ce qu'en pensent les directeurs et directrices}

\section{Collecte des données et population cible}

Dans les deux premières parties traitant de la complexité et de l'incertitude en général et dans le secteur de l'éducation en particulier, il a été mis en relief des facteurs généraux de l'incertitude, mais tout à fait valables dans le contexte québécois, tels l'éclatement du système de valeurs, le relâchement des liens sociaux, la prévalence croissante de l'individuel sur le collectif, l'accélération du changement, la pluralité ethnique et culturelle, etc. Les États généraux sur l'éducation et une dizaine d'années de réflexions et de consultations, comme le souligne le Conseil supérieur de l'éducation (1995), avaient mis en relief les multiples problèmes qui plaidaient " pour la réforme du système de l'éducation".

S'ajoutent à ces facteurs qui rendent de plus en plus incertain et complexe le monde scolaire ceux qui sont plus spécifiquement et directement reliés à la réforme elle-même et portés par elle : incertitude quant aux ressources additionnelles nécessitées par la mise en œuvre de la réforme, incertitude quant au degré de préparation des «bénéficiaires» du nouveau partage des pouvoirs, incertitude quant aux réactions en pratique des anciens détenteurs des pouvoirs redistribués, incertitude quant à l'impact des changements envisagés sur la réussite scolaire, etc.

Plusieurs catégories d'acteurs, pour ne pas dire tous les acteurs du monde scolaire, sont concernés par ces changements : commissaires et gestionnaires des commissions scolaires, directeurs et directrices d'établissements scolaires, enseignants, parents d'élèves, élèves, etc. Dans cette recherche exploratoire sur les perceptions des acteurs par rapport à la réforme, dont l'axe majeur est un transfert de pouvoir des commissions vers les établissements scolaires, nous avons arrêté notre choix sur les directeurs d'école et les commissaires et sur l'entrevue semi-structurée pour la collecte des informations.

L'instrument est en fait un bref schéma d'entretien comportant une dizaine d'énoncés de questions, la plupart ouvertes, articulées essentiellement autour des changements anticipés (ou en cours) de la réforme traduite par les modifications apportées par la loi 180 à la Loi sur l'instruction publique en relation notamment avec :

1) le pouvoir et son partage entre les commissions et les établissements scolaires;

2) le rôle et le leadership des directeurs et directrices d'établissements scolaires;

3) les nouveaux modes d'exercice des pouvoirs scolaires à travers le conseil d'établissement et la gestion participative;

4) les effets attendus de la réforme sur le fonctionnement de l'école et la réussite scolaire.

La recherche a été effectuée au cours de l'automne 1998, dans la région immédiate de Québec, auprès d'un échantillon intentionnel de 8 directeurs et 4 directrices d'écoles pour un total de 12 et de 7 commissaires. Tenant compte du caractère très important de leur rôle de leadership, dans le cadre de cet article il ne sera question que des perceptions et opinions des directeurs et directrices d'école, dont nous 
décrivons à toutes fins utiles le profil socio-démographique. Nous présentons également la fiche signalétique des établissements dont ils ont la charge. Un bref coup d'œil sur le Tableau I suivant montre un profil de directeurs d'âge mûr oscillant entre 40-45 ans et 50-55 ans.

Tableau I - Profil des chefs d'établissement interviewés

\begin{tabular}{|c|c|c|c|}
\hline Caractéristiques & Primaire & Secondaire & Total \\
\hline $\begin{array}{l}\text { Sexe : } \\
\text { - Masculin } \\
\text { - Féminin }\end{array}$ & $\begin{array}{l}4 \\
2\end{array}$ & $\begin{array}{l}4 \\
2\end{array}$ & $\begin{array}{l}8 \\
4\end{array}$ \\
\hline $\begin{array}{l}\text { Âge: } \\
-40-45 \text { ans } \\
-45-50 \text { ans } \\
-50-55 \text { ans }\end{array}$ & $\begin{array}{l}1 \\
4 \\
1\end{array}$ & $\begin{array}{l}3 \\
1 \\
2\end{array}$ & $\begin{array}{l}4 \\
5 \\
3\end{array}$ \\
\hline $\begin{array}{l}\text { Années de scolarité : } \\
\text { - } 17 \text { à moins de } 19 \text { ans } \\
\text { - } 19 \text { ans et plus }\end{array}$ & $\begin{array}{l}4 \\
2\end{array}$ & $\begin{array}{l}5 \\
1\end{array}$ & $\begin{array}{l}9 \\
3\end{array}$ \\
\hline $\begin{array}{l}\text { Expérience de direction : : } \\
\text { - Moins de } 5 \text { ans } \\
\text { - } 5 \text { à moins de } 10 \text { ans } \\
\text { - } 10 \text { ans et plus }\end{array}$ & $\begin{array}{l}1 \\
- \\
5\end{array}$ & $\begin{array}{l}1 \\
- \\
5\end{array}$ & $\begin{array}{c}2 \\
- \\
10\end{array}$ \\
\hline Total & 6 & 6 & 12 \\
\hline
\end{tabular}

Le vieillissement des personnels de l'éducation est un phénomène bien connu au Québec, avec tout ce que cela peut impliquer en termes de poids du changement et des incertitudes qui y sont associés. À cet égard, il sera intéressant et important d'étudier les réactions des divers acteurs devant la réforme scolaire par rapport à leur âge. En ce qui concerne leur formation et l'étendue de leur expérience, on se rend compte qu'ils ont un niveau élevé de scolarité (17 ans et plus) avec une longue expérience de direction d'établissements scolaires, exception faite de deux directeurs.

Concernant leurs établissements, au nombre de 12, ils se répartissent également entre le primaire et le secondaire. Pour le primaire, l'effectif-élèves se situe entre un minimum de 270 pour 16 enseignants et un maximum de 720 pour 35 enseignants. $\mathrm{Au}$ secondaire, les chiffres montent à 552 pour 35 enseignants dans le plus petit établissement et à 2500 pour 175 enseignants dans le plus grand. Soulignons pour finir qu'au primaire, à l'exception de l'établissement ayant 720 élèves, les directeurs et directrices n'ont pas d'adjoints, ce qui n'est pas le cas au secondaire où tous en ont, à un nombre se situant entre 2 pour le plus petit établissement et 7 pour le plus grand.

Au total, c'est avec beaucoup d'intérêt que les directeurs et directrices sollicités, malgré leurs lourdes tâches, ont participé à ces entrevues d'une durée moyenne d'une heure environ, apportant leurs idées, opinions et perceptions, que nous présentons et analysons plus loin. Mais, auparavant, il nous a paru utile de faire état 
même brièvement de quelques changements majeurs découlant de cette réforme, notamment en termes de redistribution des pouvoirs entre les instances et les acteurs de l'organisation scolaire.

\section{La réforme et les pouvoirs scolaires}

Relativement à l'organisation et à la gestion des services éducatifs au niveau précollégial, la loi 180 modifiant la Loi québécoise sur l'instruction publique entraîne une décentralisation des pouvoirs des commissions scolaires vers les établissements scolaires, créant une dynamique nouvelle par l'institution du conseil d'établissement et le transfert de responsabilités nouvelles au directeur (à la directrice). Le postulat sous-jacent à la réforme est que l'école, devenant plus autonome à la suite de la décentralisation des pouvoirs, sera mieux à même de réaliser sa mission d'assurer la réussite scolaire du plus grand nombre d'élèves.

Structurellement, on distingue toujours :

1. le niveau central, avec le Ministère qui élabore les politiques et définit les grandes orientations de l'éducation, voit à une juste répartition des ressources entre les commissions scolaires et à la qualité de l'éducation par la délivrance des diplômes;

2. le niveau intermédiaire, celui des commissions scolaires qui doivent s'assurer que les élèves, jeunes et adultes de leur territoire, reçoivent les services prévus par la loi et, à cette fin, répartir équitablement les ressources entre les établissements scolaires;

3. le niveau de la base, celui précisément des établissements dont le rôle consiste à dispenser les services éducatifs au élèves, dans le respect du principe d'égalité des chances, et à collaborer au développement social et culturel de la communauté.

Le grand changement apporté par la loi 180 est donc la création du conseil d'établissement (CE), nouvelle instance dotée de pouvoirs et de responsabilités traduisant une autonomisation croissante de l'école. La loi prévoit évidemment la composition et les modes de fonctionnement du conseil. À cet égard, il y a lieu de souligner la consolidation des pouvoirs des parents, dont l'un assure la présidence du conseil, la reconnaissance formelle des membres du personnel, notamment enseignant, et de leur rôle dans le fonctionnement de l'établissement ainsi que l'émergence de la communauté comme partenaire de l'école. Les élèves restent toujours au centre de l'établissement scolaire mais beaucoup plus comme bénéficiaires que comme agents de pouvoir. Les quatre tableaux suivants schématisent les fonctions et pouvoirs du conseil d'établissement parallèlement à ceux des autres acteurs qui y siègent ainsi que, en ce qui les concerne, des commissions scolaires:

1. TABLEAU II - Fonctions et pouvoirs généraux

2. TABLEAU III - Fonctions et pouvoirs en matière de services éducatifs

3. TABLEAU IV - Fonctions et pouvoirs en matière de ressources matérielles et financières

4. TABLEAU V - Autres fonctions et pouvoirs 
Un examen sommaire de ces quatre tableaux montre que le CE est le pivot de la structure de base du système scolaire, responsable de la détermination des orientations et des modalités des services éducatifs à offrir aux élèves et à la communauté, dans le cadre d'un partenariat dynamique avec la commission scolaire et le personnel de l'établissement et dans le respect des prérogatives du directeur de l'école, responsable de la gestion du personnel. Nous examinons à présent les réactions des directeurs par rapport à ces changements.

Tableau II - Fonctions et pouvoirs généraux

\begin{tabular}{|c|c|c|c|c|}
\hline & $\begin{array}{c}\text { Conseil } \\
\text { d'établissement }\end{array}$ & Directeur & $\begin{array}{c}\text { Membres } \\
\text { du personnel }\end{array}$ & $\begin{array}{l}\text { Commission } \\
\text { scolaire }\end{array}$ \\
\hline Projet éducatif & $\begin{array}{l}\text { Adopte, voit à } \\
\text { la réalisation et } \\
\text { à l'évaluation } \\
\text { (art. } 74 \text { ) }\end{array}$ & $\begin{array}{l}\text { En coordonne } \\
\text { l'élaboration, } \\
\text { la réalisation } \\
\text { et l'évaluation } \\
\text { périodique } \\
\text { (art. 96.13) }\end{array}$ & $\begin{array}{l}\text { Participent } \\
\text { (art. 74) }\end{array}$ & $\begin{array}{l}\text { En favorise } \\
\text { la réalisation } \\
\text { (art. } 218 \text { ) }\end{array}$ \\
\hline $\begin{array}{l}\text { Politique d'encadrement } \\
\text { des élèves }\end{array}$ & $\begin{array}{l}\text { Approuve } \\
\text { (art. 75) }\end{array}$ & $\begin{array}{l}\text { S'assure de } \\
\text { l'élaboration } \\
\text { (art. 96.13) }\end{array}$ & $\begin{array}{l}\text { Participent } \\
\text { (art. } 77 \text { ) }\end{array}$ & \\
\hline $\begin{array}{l}\text { Règles de conduite et } \\
\text { mesures de sécurité }\end{array}$ & $\begin{array}{l}\text { Approuve } \\
\text { (art. 76) }\end{array}$ & $\begin{array}{l}\text { Propose } \\
\text { (art. 75) }\end{array}$ & $\begin{array}{l}\text { Participent } \\
\text { (art. } 77 \text { ) }\end{array}$ & \\
\hline $\begin{array}{l}\text { Rapport annuel des } \\
\text { activités de l'école }\end{array}$ & $\begin{array}{l}\text { Prépare, adopte } \\
\text { et transmet une } \\
\text { copie à la CS } \\
\text { (art. } 82 \text { ) }\end{array}$ & & & $\begin{array}{l}\text { Prépare un } \\
\text { rapport sur les } \\
\text { activités éduca- } \\
\text { tives et cultu- } \\
\text { relles de ses } \\
\text { écoles (art. 200) }\end{array}$ \\
\hline $\begin{array}{l}\text { Services offerts } \\
\text { par l'école }\end{array}$ & $\begin{array}{l}\text { Informe la } \\
\text { communauté } \\
\text { et rend compte } \\
\text { de leur qualité } \\
\text { (art. 83) }\end{array}$ & & & \\
\hline $\begin{array}{l}\text { Modification ou } \\
\text { révocation de l'acte } \\
\text { d'établissement }\end{array}$ & $\begin{array}{l}\text { Est consulté } \\
\text { (art. 79) }\end{array}$ & & & $\begin{array}{l}\text { Consulte } \\
\text { et décide } \\
\text { (art. } 40 \text { et 217) }\end{array}$ \\
\hline $\begin{array}{l}\text { Critères de sélection } \\
\text { du directeur de l'école }\end{array}$ & $\begin{array}{l}\text { Est consulté } \\
\text { (art. 79) }\end{array}$ & & & $\begin{array}{l}\text { Consulte } \\
\text { et nomme } \\
\text { (art. } 79 \text { et 96.8) }\end{array}$ \\
\hline $\begin{array}{l}\text { Reconnaissance } \\
\text { confessionnelle de l'école }\end{array}$ & $\begin{array}{l}\text { Est consulté } \\
\text { (art. 79) }\end{array}$ & & & $\begin{array}{l}\text { Consulte et } \\
\text { donne suite } \\
\text { (art. 218) }\end{array}$ \\
\hline $\begin{array}{l}\text { Questions ou sujets rela- } \\
\text { tifs à la bonne marche de } \\
\text { l'école ou à une meilleure } \\
\text { organisation des services } \\
\text { dispensés par la CS }\end{array}$ & $\begin{array}{l}\text { Donne son } \\
\text { avis à la CS }\end{array}$ & & & \\
\hline
\end{tabular}


Tableau III - Fonctions et pouvoirs en matière de services éducatifs

\begin{tabular}{|c|c|c|c|c|}
\hline & $\begin{array}{c}\text { Conseil } \\
\text { d'établissement }\end{array}$ & Directeur & $\begin{array}{l}\text { Membres } \\
\text { du personnel }\end{array}$ & $\begin{array}{l}\text { Commission } \\
\text { scolaire }\end{array}$ \\
\hline $\begin{array}{l}\text { Modalités d'application } \\
\text { du régime pédagogique }\end{array}$ & $\begin{array}{l}\text { Approuve } \\
\text { (art. 80) }\end{array}$ & $\begin{array}{l}\text { S'assure de } \\
\text { l'élaboration } \\
\text { (art. 96.13) }\end{array}$ & $\begin{array}{l}\text { Participent } \\
\text { (art. 89) }\end{array}$ & $\begin{array}{l}\text { S'assure de } \\
\text { l'application } \\
\text { du régime } \\
\text { pédagogique } \\
\text { (art. 222) }\end{array}$ \\
\hline $\begin{array}{l}\text { Orientations générales en } \\
\text { matière d'enrichissement } \\
\text { et d'adaptation des } \\
\text { programmes }\end{array}$ & $\begin{array}{l}\text { Approuve } \\
\text { (art. 85) }\end{array}$ & $\begin{array}{l}\text { S'assure de } \\
\text { l'élaboration } \\
\text { (art. 96.13) } \\
\text { Propose } \\
\text { (art. 85) }\end{array}$ & $\begin{array}{l}\text { Participent (**) } \\
\text { (art. 89) }\end{array}$ & $\begin{array}{l}\text { S'assure de } \\
\text { la mise en } \\
\text { œuvre des } \\
\text { programmes } \\
\text { (art. 222.1) }\end{array}$ \\
\hline $\begin{array}{l}\text { Temps alloué à } \\
\text { chaque matière }\end{array}$ & $\begin{array}{l}\text { Approuve } \\
\text { (art. 86) }\end{array}$ & $\begin{array}{l}\text { S'assure de } \\
\text { l'élaboration } \\
\text { (art. 96.13) } \\
\text { Propose } \\
\text { (art. 85) }\end{array}$ & $\begin{array}{l}\text { Participent }(* *) \\
\text { (art. 89) }\end{array}$ & \\
\hline $\begin{array}{l}\text { Programmes d'études } \\
\text { locaux }\end{array}$ & Est informé & $\begin{array}{l}\text { Approuve } \\
\text { (art. 96.15) }\end{array}$ & $\begin{array}{l}\text { Proposent }\left({ }^{*}\right) \\
\text { (art. 96.15) }\end{array}$ & \\
\hline $\begin{array}{l}\text { Critères relatifs à } \\
\text { l'implantation de } \\
\text { nouvelles méthodes } \\
\text { pédagogiques }\end{array}$ & Est informé & $\begin{array}{l}\text { Approuve } \\
\text { (art. 96.15) }\end{array}$ & $\begin{array}{l}\text { Proposent }(* *) \\
\text { (art. 96.15) }\end{array}$ & $\begin{array}{l}\text { S'assure que } \\
\text { l'école ne se } \\
\text { sert que } \\
\text { des manuels } \\
\text { et du matériel } \\
\text { approuvés } \\
\text { (art. 230) }\end{array}$ \\
\hline $\begin{array}{l}\text { Choix des manuels } \\
\text { scolaires et du matériel } \\
\text { didactique }\end{array}$ & Est informé & $\begin{array}{l}\text { Consulte le CE } \\
\text { et approuve } \\
\text { (art. 96.15) }\end{array}$ & $\begin{array}{l}\text { Proposent }(* *) \\
\text { (art. 96.15) }\end{array}$ & \\
\hline $\begin{array}{l}\text { Programmation des } \\
\text { activités éducatives qui } \\
\text { nécessitent un change- } \\
\text { ment aux heures d'entrée } \\
\text { et de sortie quotidienne } \\
\text { des élèves ou un } \\
\text { déplacement à l'extérieur } \\
\text { des locaux de l'école }\end{array}$ & $\begin{array}{l}\text { Approuve } \\
\text { (art. 87) }\end{array}$ & $\begin{array}{l}\text { S'assure de } \\
\text { l'élaboration } \\
\text { (art. 96.13) } \\
\text { Propose } \\
\text { (art. 87) }\end{array}$ & $\begin{array}{l}\text { Participent } \\
\text { (art. 89) }\end{array}$ & \\
\hline $\begin{array}{l}\text { Mise en œuvre des } \\
\text { programmes des services } \\
\text { complémentaires et } \\
\text { particuliers }\end{array}$ & $\begin{array}{l}\text { Approuve } \\
\text { (art. 88) }\end{array}$ & $\begin{array}{l}\text { S'assure de } \\
\text { l'élaboration } \\
\text { (art. 96.13) } \\
\text { Propose } \\
\text { (art. 85) }\end{array}$ & $\begin{array}{l}\text { Participent } \\
\text { (art. 89) }\end{array}$ & $\begin{array}{l}\text { Établit les } \\
\text { programmes } \\
\text { (art. 224) }\end{array}$ \\
\hline $\begin{array}{l}\text { Critères d'inscription } \\
\text { des élèves }\end{array}$ & $\begin{array}{l}\text { Est informé } \\
\text { (art. 239) }\end{array}$ & & & $\begin{array}{l}\text { Transmet au CE } \\
15 \text { jours avant } \\
\text { le début de } \\
\text { la période } \\
\text { d'inscription } \\
\text { (art. 239) }\end{array}$ \\
\hline $\begin{array}{l}\text { Enseignement moral } \\
\text { et religieux autre que } \\
\text { catholique ou protestant }\end{array}$ & $\begin{array}{l}\text { Peut demander } \\
\text { (art. 228) }\end{array}$ & & & $\begin{array}{l}\text { Peut organiser } \\
\text { (art. 228) }\end{array}$ \\
\hline
\end{tabular}




\begin{tabular}{|l|l|l|l|l|}
\hline $\begin{array}{l}\text { Normes et modalités } \\
\text { d'évaluation }\end{array}$ & Est informé & $\begin{array}{l}\text { Approuve } \\
\text { (art. 96.15) }\end{array}$ & $\begin{array}{l}\text { Proposent }{ }^{* *} \text { ) } \\
\text { (art. 96.15) }\end{array}$ & $\begin{array}{l}\text { S'assure que } \\
\text { l'école évalue } \\
\text { les apprentis- } \\
\text { sages et } \\
\text { applique les } \\
\text { épreuves } \\
\text { imposées par } \\
\text { le ministre } \\
\text { (art. 231) } \\
\text { Peut imposer } \\
\text { des épreuves } \\
\text { internes } \\
\text { (art. 233) }\end{array}$ \\
\hline $\begin{array}{l}\text { Règles pour le classe- } \\
\text { ment des élèves et } \\
\text { passage d'un cycle à } \\
\text { l'autre au primaire }\end{array}$ & Est informé & $\begin{array}{l}\text { Approuve } \\
\text { (art. 96.15) }\end{array}$ & $\begin{array}{l}\text { Proposent } \\
\text { (art. 96.15) }\end{array}$ & $\begin{array}{l}\text { Établit les règles } \\
\text { pour le passage } \\
\text { du primaire au } \\
\text { secondaire et } \\
\text { du 1er et 2e } \\
\text { cycle du secon- } \\
\text { daire (art. 230) }\end{array}$ \\
\hline
\end{tabular}

(*i) Personnel enseignant seulement.

Tableau IV - Fonctions et pouvoirs en matière de ressources matérielles et financières

\begin{tabular}{|c|c|c|c|c|}
\hline & $\begin{array}{c}\text { Conseil } \\
\text { d'établissement }\end{array}$ & Directeur & $\begin{array}{c}\text { Membres } \\
\text { du personnel }\end{array}$ & $\begin{array}{l}\text { Commission } \\
\text { scolaire }\end{array}$ \\
\hline Utilisation des locaux & $\begin{array}{l}\text { Approuve } \\
\text { (art. 93) }\end{array}$ & $\begin{array}{l}\text { Propose } \\
\text { (art. 93) }\end{array}$ & & $\begin{array}{l}\text { Autorise si } \\
\text { entente de } \\
\text { plus d'un an } \\
\text { (art. 93) }\end{array}$ \\
\hline Dons et contributions & $\begin{array}{l}\text { Peut solliciter } \\
\text { et recevoir un } \\
\text { don ou une con- } \\
\text { tribution et sur- } \\
\text { veille l'adminis- } \\
\text { tration du fonds } \\
\text { (art. 94) }\end{array}$ & & & $\begin{array}{l}\text { Crée un fonds } \\
\text { à destination } \\
\text { spéciale, tient } \\
\text { des livres et } \\
\text { comptes séparés } \\
\text { (art. 94) }\end{array}$ \\
\hline Budget annuel de l'école & $\begin{array}{l}\text { Adopte } \\
\text { (art. 95) }\end{array}$ & $\begin{array}{l}\text { Prépare } \\
\text { (art. 96.24) }\end{array}$ & & $\begin{array}{l}\text { Répartit les } \\
\text { ressources entre } \\
\text { les écoles } \\
\text { (art. 276) }\end{array}$ \\
\hline $\begin{array}{l}\text { Besoins de l'école relatifs } \\
\text { aux biens et services et } \\
\text { aux locaux }\end{array}$ & $\begin{array}{l}\text { Est consulté } \\
\text { (art. 96.22) }\end{array}$ & $\begin{array}{l}\text { Consulte le CE } \\
\text { et fait part à la } \\
\text { CS des besoins } \\
\text { (art. 96.22) }\end{array}$ & & \\
\hline
\end{tabular}


Tableau $V$ - autres fonctions et pouvoirs

\begin{tabular}{|c|c|c|c|c|}
\hline & $\begin{array}{c}\text { Conseil } \\
\text { d'établissement }\end{array}$ & Directeur & $\begin{array}{c}\text { Membres } \\
\text { du personnel }\end{array}$ & $\begin{array}{l}\text { Commission } \\
\text { scolaire }\end{array}$ \\
\hline Services extrascolaires & $\begin{array}{l}\text { Peut organiser } \\
\text { (art. 74) } \\
\text { Peut conclure } \\
\text { un contrat ("*) } \\
\text { et doit le trans- } \\
\text { mettre à la CS } \\
\text { (art. 91) }\end{array}$ & & & $\begin{array}{l}\text { Peut indiquer } \\
\text { son désaccord } \\
\text { (art. 91) }\end{array}$ \\
\hline Surveillance du midi & $\begin{array}{l}\text { Convient des } \\
\text { modalités avec } \\
\text { la CS } \\
\text { (art. 292) }\end{array}$ & & & $\begin{array}{l}\text { Assure la sur- } \\
\text { veillance du } \\
\text { midi selon les } \\
\text { modalités con- } \\
\text { venues avec les } \\
\text { CE et aux condi- } \\
\text { tions financières } \\
\text { qu'elle peut } \\
\text { déterminer } \\
\text { (art. 292) }\end{array}$ \\
\hline $\begin{array}{l}\text { Services de garde en } \\
\text { milieu scolaire }\end{array}$ & $\begin{array}{l}\text { Peut demander } \\
\text { (art. 256) }\end{array}$ & & & $\begin{array}{l}\text { Doit assurer des } \\
\text { services de garde } \\
\text { (art. 256) }\end{array}$ \\
\hline
\end{tabular}

(") Au nom de la commission scolaire

\section{Perceptions et réactions des directeurs devant les changements liés à la loi 180}

La présentation et l'analyse des données seront articulées autour de deux points : dans un premier temps, il sera question des incertitudes liées aux changements apportés par la loi 180; et dans un deuxième temps de l'impact anticipé de la réforme sur le fonctionnement de l'école et la réussite scolaire.

\section{Changements et incertitudes}

a. Programmes d'études et curriculum

Depuis plusieurs années, soulignent plusieurs directeurs, le réseau d'éducation vit des modifications substantielles. Une façon de dire que la loi 180 n'est qu'un aboutissement d'un long processus, où, pour reprendre les propos ternes d'un interviewé :

"Ce qui inquiète particulièrement est d'en arriver encore à d'autres substitutions de programmes, donc des modifications en termes de contenus (curriculum), quand on n'a pas encore terminé, notamment la formation des maîtres, sur ce qui déjà précédait. »

Cette inquiétude relative aux programmes, au curriculum et au régime pédagogique est partagée par la plupart des directeurs. D'un côté, ils disent «ne pas avoir beaucoup d'informations pour bien comprendre» et, de l'autre, certains se 
voient déjà pris «entre l'arbre et l'écorce», entre «le Conseil d'établissements sans l'aspect pédagogique et le Directeur général de la Commission scolaire, en termes de gestion» (sic). «Beaucoup d'enseignants, soulignent-ils, se sentent vulnérables et auront besoin de se recycler en fonction des nouveaux programmes d'études. » Cela crée beaucoup d'appréhensions. Mais, pour l'ensemble des interviewés, l'axe central de la réforme, c'est le nouveau partage des responsabilités et des pouvoirs. Comment perçoivent-ils les changements en ce sens?

b. Pouvoirs et responsabilités : une dynamique nouvelle

De l'avis de tous, le changement le plus important, c'est la prise en charge de l'école par le milieu, les parents, le personnel, le personnel enseignant notamment, le directeur demeurant le leader avec des tâches supplémentaires et une valorisation accrue. Ils sont, dans l'ensemble, conscients cependant que si on peut parler de décentralisation quand il s'agit du projet éducatif, pour plusieurs autres objets de gestion, ce n'est que de la déconcentration, "les commissions scolaires ayant encore leur mot à dire, ayant encore des leviers de contrôle, des critères et des exigences ». Par rapport à ce point, presque tous les participants, tout en étant modérément optimistes, ont exprimé quelques inquiétudes :

- d'abord que les responsabilités dévolues à la base ne soient pas accompagnées des pouvoirs et surtout des ressources budgétaires nécessaires;

- en deuxième lieu, que les autorités des commissions scolaires utilisent les leviers entre leurs mains pour freiner sinon bloquer la mise en application de la réforme.

Par ailleurs, la prise en charge de l'école par l'ensemble de ses acteurs, via le conseil d'établissement, entraînera l'émergence d'une pluralité de visions et de logiques différentes d'action, la création de nouveaux rapports de force, voire de contradictions. Il est certain que, sur les problèmes de l'école, les enseignants n'auront pas toujours le même point de vue que les parents, les représentants du milieu ou les directions d'école et réciproquement. Ainsi, le pouvoir, tel que prévu par la loi dans sa définition et sa répartition entre les acteurs, sera souvent contrebalancé (ou contrecarré) par le pouvoir d'influence que donnent notamment la détention de l'information et la connaissance des problèmes. Nous pensons ici au pouvoir d'expertise des directeurs d'école et des enseignants. Ces réflexions du chercheur sont validées par les répondants. Certes, s'ils reconnaissent unanimement les nouveaux pouvoirs et responsabilités de l'établissement comme une bonne chose pour permettre de rejoindre les véritables besoins des élèves, ils n'expriment pas moins plusieurs préoccupations allant dans ce sens :

1. Concernant le degré de préparation des différents acteurs pour bien jouer leur nouvelle partition, écoutons les propos de ce directeur parlant des parents : "J'suis pas sûr que l'ensemble des parents du Québec souhaitaient qu'on aille aussi loin et j'suis pas sûr que l'ensemble des parents du Québec sont prêts à en assumer autant.» 
"C'est la même chose pour les enseignants, eux qui réclament depuis longtemps plus d'autonomie professionnelle, d'être partie prenante aux décisions, sauf que là, ça arrive... Et c'est pas toujours facile.»

Ils ont également des inquiétudes concernant eux-mêmes, les directeurs et directrices, piliers de la réforme. Voici comment l'un d'entre eux s'exprime à ce sujet :

«Il y a des directeurs d'école qui vont trouver ça très difficile. Parce que tous ceux qui n'ont pas une approche de participation, il va falloir qu'ils changent complètement. Il y en a qui sont pas capables; ils devront donc s'en aller.»

Les directeurs d'école sont donc très conscients de l'importance stratégique de leur rôle et de leurs fonctions, pour sensibiliser et informer les parents et la communauté en général et assurer un climat de confiance et de coopération entre les divers intervenants.

À cet égard, ils savent, comme le souligne l'un d'eux:

" ... faut quand même pas oublier que les parents, quand ils vont là (au $\mathrm{CE})$, pensent en tant que parents, que les enseignants pensent en tant qu'enseignants et que le directeur pense en tant qu'administrateur de l'école».

Cela dit, ils sont confiants, dans l'ensemble, qu'ils vont apprendre sur le tas et que

«ces inquiétudes-là, de part et d'autre, vont disparaître et assez rapidement.»

2. Inquiétude relative aux conflits possibles entre les acteurs par rapport aux pouvoirs

D'emblée, tous les intervenants sont unanimes à reconnaître que le transfert des pouvoirs vers l'établissement scolaire est une excellente chose. Certains soulignent qu'il en découlera une meilleure identification de l'école à son environnement, un plus grand engagement et intérêt des acteurs de l'école et de la communauté, plus globalement, pour les problèmes de l'école. Mais des craintes sont vivement exprimées concernant le partage et l'exercice de ces pouvoirs et les possibilités réelles de forger les consensus.

Ainsi, l'un des directeurs s'inquiète «qu'un peu tout le monde s'empiète (sic) l'un sur l'autre et c'est sûr, poursuit-il, qu'au début, il va y avoir des accrochages ». Dans le même ordre d'idées, souligne un autre, y allant encore plus systématiquement:

«pour les parents, ils sont inquiets que leurs responsabilités ne viennent pas avec les pouvoirs et les ressources nécessaires, alors que l'établissement est imputable quand ça ne marche pas au niveau de 
l'école, on ne pourra plus dire: c'est la Commission scolaire, ce n'est pas nous autres".

À cet égard, il faut parler d'une troisième source spécifique d'incertitude et d'inquiétudes.

3. Inquiétudes relatives à une surcharge des directeurs et directrices d'école Il a déjà été évoqué précédemment que l'un des changements associé à la loi 180 est la charge supplémentaire de travail qu'elle entraîne pour les directeurs, pas seulement en termes quantitatifs mais aussi en termes qualitatifs.

À ce sujet, un des interviewés s'exprime comme suit :

«De plus en plus de directeurs souffrent de 'burnout', de stress, de dépression et de différents problèmes d'ordre physique. C'est pas pour rien ça... Tout est à peu près de la responsabilité de l'école. Oui, je vous dis que la tâche de direction d'école s'est alourdie."

Par ailleurs, on a certes toujours considéré le directeur comme le leader, celui ou celle qui coordonne l'ensemble des troupes. L'aspect politique de la fonction augmente dans le contexte de la réforme, comme le souligne cet intervenant:

«Il y a toute la dimension politique maintenant qui fait partie de l'environnement du directeur d'école... mais aussi des enseignants. Le directeur d'école avait déjà, avec les conseils d'orientation, une certaine activité politique. Mais là, c'est un conseil d'administration et la partie la plus importante du conseil d'établissement, c'est les enseignants. Et au lieu de s'opposer, ils devront établir avec les parents, une complicité. Cette complicité-là est politique en plus. »

Cette observation sur le changement qualitatif de la tâche des directeurs et les nouveaux rapports de force nous amène à parler d'une autre source importante d'incertitude pour eux.

4. Syndicalisme versus professionnalisme

Pour la plupart des directeurs interviewés, au-delà du transfert des pouvoirs vers l'école, l'un des changements majeurs qu'apporte la loi 180, c'est la consécration de l'autonomie professionnelle de l'enseignant. La rénovation des programmes et des curricula, comme dit un directeur, appelle « une mobilisation générale des enseignants. Mais les enseignants, depuis plusieurs années, ont été plus mobilisés par l'aspect syndical que par la démarche de l'école.» On peut traduire ce propos en disant que la réforme scolaire invite les enseignants à un véritable virage culturel : soit d'une culture syndicale de caractère global, axée sur les intérêts des membres, à une culture professionnelle orientée vers les besoins des élèves et de l'établissement scolaire. Personne d'entre les directeurs interviewés n'a mis en doute l'importance majeure des enseignants dans cette réforme. Personne d'entre eux n'a considéré comme 
incompatible le fait d'être le membre d'un corps ayant des intérêts propres et le fait d'être un professionnel capable de se dédier à ses fonctions et à ses tâches. Mais l'inquiétude transpire, quand certains directeurs disent:

«Il y a plusieurs enseignants qui, dans le fond, en sont rendus à se foutre carrément de tous les changements qui sont annoncés et qu'on verra ce qu'on fera quand on sera rendu là... Parce qu'on est dans une phase de négociation de convention collective... et que les enseignants, sous un prétexte syndical, se retirent carrément de la démarche.»

Incertitude liée donc à un changement de culture que semble appeler la réforme et à un événement conjoncturel (une négociation collective) dont il est difficile de prévoir les issues. Toutes ces incertitudes ne sont peut-être pas sans lien avec les perceptions des directeurs quant aux effets attendus de la réforme scolaire.

\section{Effets attendus de la réforme et conditions de réalisation}

Au départ, il y a lieu de dire que la loi 180 est complémentaire du plan d'action ministériel pour la réforme de l'éducation, au sujet duquel madame Pauline Marois, dans la présentation du 24 août 1996 des grandes orientations, soulignait l'urgence de « donner un important coup de barre afin de passer de l'accès du plus grand nombre, au succès du plus grand nombre ", l'un des moyens privilégiés par la loi étant " la décentralisation des pouvoirs pour donner plus d'autonomie à l'école ».

Par rapport à la typologie des cas d'incertitudes esquissée précédemment, nous nous situons dans les deux derniers cas, où le degré d'information et la marge de manœuvre et d'autonomie des acteurs s'avèrent relativement élevés.

Par rapport à l'objectif visé, nous avons amené les directeurs à donner leurs points de vue quant aux effets de la réforme pour le fonctionnement de l'école et la réussite scolaire des élèves.

Dans l'ensemble, les directeurs et directrices interviewés se disent modérément ou très optimistes, confiants que «les gens vont embarquer " dans la mise en œuvre (l'application) de cette loi qui, de l'avis de la plupart, « est une très belle loi,... qui a vraiment ciblé les bons responsables, donnant, en ce qui concerne les aspects pédagogiques, le pouvoir aux écoles». Pour l'un des directeurs, son optimisme est lié au fait que «ça faisait plusieurs années que le virage était amorcé, à travers en particulier les conseils d'orientation». Donc, pour lui, «l'ensemble des écoles et l'ensemble des directions d'école de la Commission scolaire sont équipées et prêtes pour prendre le fameux virage proposé au Québec par madame Marois». Il est vrai, en un sens, que la réforme scolaire se situe dans la continuité de nombre de mesures, au plan administratif et pédagogique, qui avaient marqué le fonctionnement des établissements scolaires: les conseils d'orientation et les comités de parents visaient déjà la participation de la «base» à la gestion de l'école. De même, le "projet éducatif", avec la préoccupation pour les aspects pédagogiques, avait fait son entrée depuis belle lurette dans la vie des établissements scolaires. 
Mais avec l'actuelle réforme, ces choses sont désormais systématisées et encadrées législativement. La plupart des interviewés soulignent que «le centre de décisions étant près des gens, il sera plus facile de prendre des décisions en fonction des besoins des communautés et des élèves et que leur réussite va être favorisée ».

Dans la même veine, un autre directeur dit que «c'est une réforme qui l'enchante et le motive, particulièrement au niveau du projet éducatif; là [il pense qu']il s'agit d'une véritable décentralisation".

Mais tous sont d'avis, comme l'a souligné l'un d'entre eux, que «c'est à nous autres à travailler avec l'équipe-école au niveau du développement; c'est à nous autres à travailler au niveau du Conseil d'établissement pour favoriser une orientation claire qui puisse aussi s'appliquer ».

Optimistes quant aux effets attendus de la réforme sur l'amélioration du fonctionnement de l'école, plus collée aux réalités et aux valeurs du milieu, sur l'amélioration du vécu et de la réussite scolaire des élèves, les interviewés y mettent toutefois plusieurs conditions dont les trois plus importantes sont les suivantes:

a. La première condition, c'est que les gens s'impliquent. Je reprends volontiers les propres termes d'un directeur:

"Ça va faire partie des défis des directions d'école justement d'essayer de s'assurer que le maximum de personnes s'impliquent pour que ça soit une réussite.»

Ce défi qui s'impose au leadership des directeurs d'école implique que ces derniers disposent de pouvoirs réels. À l'égard de la mobilisation des acteurs de l'école, nous avons souligné précédemment les craintes exprimées par plusieurs des interviewés quant à un certain esprit de militantisme syndical qui pourrait être nuisible.

Cela dit, ils sont quasi unanimes à reconnaître que la réforme entraîne « des changements de fond, dans le sens que l'école est imputable et qu'elle récupère beaucoup [...] de responsabilités tant au niveau de la gestion pédagogique d'école que de la gestion administrative des budgets, ainsi de suite... ».

Ces observations nous amènent au cœur de la deuxième condition, et peut-être la plus importante, celle des ressources au niveau des gestionnaires de l'école.

b. Une deuxième condition du succès de la réforme, est que «les budgets, les ressources soient disponibles aux écoles et qu'ils (les autres niveaux de pouvoirs) laissent une marge de manœuvre afin de faire un véritable choix", souligne l'un des interviewés. Cet avis revient chez presque tous.

Mais au-delà de ce consensus sur l'importance des ressources, comment les directeurs d'école perçoivent-ils la situation découlant de la réforme par rapport à ce facteur? À cet égard, leurs points de vue s'articulent autour de deux axes: celui du transfert des ressources aux écoles et celui de la gestion de ces ressources.

1. En ce qui concerne la disponibilité des ressources, pour la plupart d'entre eux, la réforme reste au niveau d'une déconcentration des pouvoirs, c'està-dire à un transfert des responsabilités et de tâches mais sans l'accompa- 
gnement de ressources adéquates.

C'est ce que sous-entend ce directeur quand il souligne que :

«tous les pouvoirs actuels qui appartiennent à la commission scolaire et qui n'ont pas d'incidence monétaire, ils sont très empressés à l'heure actuelle de les délester du côté des écoles ».

Ou du moins, ce qu'ils ont pu observer les laisse dans l'incertitude, dans le conditionnel, comme ils disent.

Les propos de cette directrice d'école ne laissent pas de doute là-dessus : "ce qui m’a le plus inquiétée quand on a eu cette loi-là, je me suis dit, [...] c'est bien beau donner des pouvoirs aux écoles, mais le vrai pouvoir, c'est l'argent ». [...] «l'incertitude plus présente, c'est les modalités de subvention aux écoles pour assurer du soutien aux enseignants pour appliquer ce changement-là [...] Et on est toujours dans le conditionnel... c'est toujours les "comment". Comment ça va se faire? Comment les ressources arrivent aux écoles?»

Voyons donc, à la suite de ce commentaire, comment les répondants perçoivent les nouvelles conditions d'exercice des pouvoirs relatifs à la gestion des ressources.

2. Pouvoirs relatifs à la gestion des ressources.

En même temps que la plupart des répondants expriment le souhait d'avoir des ressources adéquates par rapport aux nouvelles responsabilités de l'école, ils sont conscients que les charges administratives pourraient les amener à négliger ou à accorder moins de temps à la mission éducative de l'école, à la réussite scolaire des élèves.

Une répondante a mis ce point en relief:

«Il y a des tâches quotidiennes que j'aimerais pas gérer comme directrice d'école, parce que ma mission première, moi, je pense qu'elle se situe au cœur de cette réforme-là, au niveau du contrat social qu'on a avec les enfants puis l'évolution du système éducatif.»

Un autre répond, soulignant qu'il voyait au début la réforme apporter beaucoup de tâches administratives, exprime une certaine crainte : « $\mathrm{Si}$ on se perd dans les dédales administratives (sic) à notre niveau, on va manquer le train...»

La plupart des répondants soulignent par ailleurs que leurs nouvelles responsabilités en matière de gestion exigent une certaine forme d'appui ou de perfectionnement, reconnaissant qu'ils n'y étaient pas nécessairement préparés. Ainsi, opine ce directeur : 
«C'est nouveau! Ça va dans certains cas demander du soutien, du ressourcement, de l'accompagnement, tout dépendant des individus. Mais cette dimension-là, est à mon avis, une dimension majeure sur ce qui s'en vient.»

À cet égard, selon eux, les directeurs d'écoles primaires pourraient avoir plus de problèmes, parce qu'ils sont le plus souvent seuls pour s'occuper de tout. C'est ce qui se dégage de ces propos de plusieurs répondants: "Les écoles primaires sont plus inquiètes en regard de ça. Évidemment que les écoles secondaires, ça s'explique [...], nous autres, on a des adjoints. Eux autres n'ont pas nécessairement d'adjoints. »

Besoin donc d'appui, de ressources et pas seulement pour eux les gestionnaires, comme le souligne un autre directeur:

«Autant nos enseignants ont besoin d'avoir des ressources autour d'eux, autant nous autres on a besoin de ressources autour de nous, puis de soutien.»

Mais la disponibilité des ressources humaines, matérielles et financières n'est pas tout. Une autre ressource que l'on est porté à négliger, c'est le temps. Nous l'examinerons séparément dans la troisième et dernière condition.

c. La troisième condition, c'est d'y aller graduellement dans la mise en œuvre de ces changements. C'est que, comme l'exprime bien un interviewé :

"Ces changements-là, on n’a pas nécessairement besoin de les faire tous la première année. Je pense, poursuit-il, qu'il y aura un échéancier à fixer, des sujets prioritaires à aborder d'année en année.»

et

"S'il y a des problèmes, comme on peut s'y attendre, surtout au début, être capable de dédramatiser.»

Cette observation exprimée par ce répondant traduit une préoccupation que nous avons eu l'occasion d'entendre de multiples fois, de manière formelle, par des associations représentant des directeurs d'école, des commissions scolaires et des enseignants. Elle correspond d'ailleurs aux principes sous-jacents aux modèles de type gradualiste préconisés par nombre de spécialistes en gestion du changement et de l'innovation.

À cet égard, les points de vue exprimés par l'ensemble des répondants soulignent que le temps est une condition importante du succès de la réforme et qu'ils ont des inquiétudes par rapport à ce facteur, comme il ressort de ces propos de cette directrice d'école:

«Changement oui, il doit y en avoir, mais de façon plus pondérée, de 
façon moins expéditive en respectant les gens qui sont dans le réseau [...]. Souvent dans notre réseau, on a tendance à vouloir faire des changements de façon très expéditive sans tenir compte des ressources humaines qui sont là.»

À propos du temps comme des autres ressources, les répondants ont souligné la nécessité de tenir compte de la diversité des milieux et de ne pas imposer, à tous sans distinction, un modèle unique.

Comme le font ressortir ces propos d'un interviewé :

"C'est pas vrai que les besoins des écoles et des élèves sont les mêmes partout. Il n'y en a pas de solution mur à mur. Il y a trop de différences entre les milieux..., trop de différences entre les communautés... Alors dans ce sens-là, plus le centre de décision est près des gens, plus on va prendre des décisions en fonction des besoins des communautés et des élèves...»

Une large convergence se dégage de l'ensemble des points de vue exprimés à cet égard quant à l'importance du projet éducatifqui devrait permettre :

«que les milieux se prennent en charge davantage, que les décisions concernant les orientations pédagogiques des écoles se prennent à la base..."

Ces conditions ne font que tempérer l'optimisme des directeurs, en ce qui concerne la possibilité somme toute réelle que la réforme atteigne ses objectifs. À cet égard, certains interviewés ont exprimé une légère inquiétude quant à un possible effet pervers de la réforme, à l'encontre de la démocratisation de l'enseignement, cette fois sous l'angle du succès :

«On va peut-être créer des disparités, souligne un directeur. Et si on pense à accessibilité, égalité des services, tout le concept de démocratisation de l'enseignement, oui il y a des risques à cet égard-là!»

Et pour un autre, dans le même ordre d'idées:

«Il y a des écoles qui vont se démarquer par leur grande qualité, par leur grand projet éducatif et d'autres qui vont se démarquer par une formation que les gens vont juger inadéquate».

Mais, souligne le premier :

"Il n'y a pas un milieu, même démuni, qui pourrait imaginer d'accepter d'avoir des écoles au rabais; il faut donc faire confiance aux gens, faire confiance aux milieux!» 


\section{Conclusion}

Les derniers mots de ce directeur traduisent bien la tendance générale des répondants face aux incertitudes liées aux changements suscités par la loi 180.

Parti de la notion d'incertitude, associée à la limitation de nos savoirs et à l'imprévisibilité du futur et du changement, conscient de la complexité de la gestion en éducation par suite des antagonismes et des contradictions qui sévissent aujourd'hui dans la société en général et les organisations scolaires en particulier, il y avait lieu pour nous d'être sceptique sinon pessimiste quant au projet de réforme scolaire au Québec.

Nous rejoignions en cela plusieurs chercheurs qui, à la suite de leur examen de certains projets de réforme scolaire aux États-Unis, concluaient à leur faible impact parce que ne débouchant pas sur une participation significative aux décisions (Malen et Ogawa, 1988), ou entrainant plutôt une collégialité artificielle (Hargreaves, 1994). Ce qui faisait dire à Gary Anderson (1998, p. 572) qu' « il y a plusieurs signes que le mouvement de réforme de l'éducation basée sur la participation traversait des problèmes sérieux".

Ce qui se dégage de notre étude, c'est que, même s'ils ne sont pas nécessairement représentatifs, les 12 directeurs interviewés confirment qu'il y a lieu d'être attentif à plusieurs objets d'incertitude et d'inquiétude, mais que l'on peut être confiant que la réforme, envisagée et déjà en cours, pourra atteindre ses principaux objectifs. Elle arrive, selon eux, à un bon moment et suite à une vaste consultation, dans un contexte, où plusieurs changements significatifs avaient déjà pris place dans la structure et l'évolution de la gouvernance de l'école. Nous sommes, comme dirait Pauline Lipman (1997, p. 5), dans un contexte spécifique où il y a des signaux que les enseignants, en conjonction avec les parents, les administrateurs et les élèves, semblent avoir une réelle opportunité de façonner des changements significatifs à l'école.

À cet égard, ils souhaitent, de manière unanime, que la réforme amène un transfert de pouvoirs réels vers les établissements et leurs acteurs. Leur inquiétude concerne ici l'engagement des enseignants et de leurs syndicats mais aussi la volonté des commissions scolaires de jouer franc jeu dans ces processus de transformation qui nécessitent de réels pouvoirs et des ressources concrètes au niveau de l'école, du directeur d'école et du conseil d'établissement. Cela ne va pas sans l'émergence de nouveaux rapports entre l'école et la commission scolaire. Mais aussi, comme ils l'ont signalé à plusieurs reprises, de nouveaux rapports de force entre les différents acteurs représentés au sein du conseil d'établissement, avec le risque de tensions sourdes sinon de conflits ouverts.

Heureusement, l'ensemble des directeurs interviewés ont montré qu'ils avaient une vision assez claire et favorable des changements requis et annoncés, qu'ils étaient conscients de l'importance de leur rôle dans l'implantation de ces changements. Si sur le plan rationnel il est très difficile de réduire l'incertitude liée à ces changements, ils sont confiants que, par l'information, la communication et le partage, ils pourront amener leurs vis-à-vis à comprendre et bien interpréter ces 
changements, à y croire et à être suffisamment motivés pour agir ensemble. Il y a là les ingrédients d'un modèle que certains spécialistes en management des ressources humaines n’ont pas hésité à recommander déjà pour «mobiliser les employés dans un climat d'incertitude» (Insight Information Inc., 1995). Sûrement que ce modèle, qui fait la promotion de la gestion participative, donc d'un partage des pouvoirs, pourra être utile dans le prolongement de cette première étape exploratoire de cette recherche qu'il importera d'élargir à un échantillon plus vaste et représentatif des directeurs d'établissements scolaires du Québec, auquel pourront être ajoutées d'autres catégories de gestionnaires impliqués dans la réforme scolaire.

\section{Références bibliographiques}

ANDERSON, G.L. (1998). Toward authentic participation: deconstructing the discourses of participatory reforms in education. In American Educational Research Journal, vol. 35, nº 4 .

BARDOT, O. (1996). Stratégies d'incertitude, dans B. Cova et J. Adkam, Paris : Economica.

BAREL, Y. (1971). Prospective et analyse de systèmes, coll. TRP, documentation française, Paris.

BOURGEAULT (1985). Turbulence. In Encyclopedia Universalis, Corpus, nº 18.

BOURY, P. (1996). Travailler en univers incertain : le commentaire du banquier. In Stratégies d'incertitude, B. Cova et J. Wickham, Paris : Economica.

BRONNER, G. (1997). L'incertitude, Collection Que Sais-je?, Paris : PUF.

BURNS, L.M. (1995). Values based planning for quality education. Lancaster-Basel, Technonic Publishing Co.

Conseil supérieur de l'éducation (1993). La gestion de l'éducation, nécessité d'un autre modèle. Rapport annuel 1991-1992 sur l'état et les besoins de l'éducation, Québec.

Conseil supérieur de l'éducation (1995). Pour la réforme du système éducatif: Dix années de consultation et de réflexion, Québec.

COVA, B., WICKHAM (1996). Stratégies d'incertitude, Paris : Economica.

GODIN, André (2000). Diriger dans les eaux vives d'un établissement en projet. Modèle descriptif de projet éducatif. (Synthèse de plusieurs communications). Texte inédit.

Gouvernement du Québec (1998). Lois-180 (1997, chapitre 96) modificant la Loi sur l'instruction publique, Ministère de l'Éducation du Québec (MEQ). 
HARGREAVES, A. (1994). Changing teachers, changing times : Teachers' work and culture in the postmodern age. New York: Teachers College Press.

HOUSE, E.R. (1996). A framework for appraising educational reforms. In Educational Researcher, vol. 25, $\mathrm{n}^{0} 7$.

Insight Information Inc. (1995). Réussir à mobiliser vos employés dans un climat d'incertitude. Textes des Conférences d'I.I.I., tenues à Montréal le 30 octobre 1995. Toronto : Insight Press.

LIPMAN, P. (1997). Restructuring in context : a case study of teacher participation in the dynamics of ideology, race and power. In American Educational Research Journal, vol. $34, \mathrm{n}^{0} 1$.

MALEN, B., OGAWA, R. (1988). Professional-patron influence on site-based governance councils : a confounding case study. In Educational Evaluation and Policy Analysis, vol. 10, n 4.

MENGER, M. (1991). Marché du travail artistique et socialisation du risque. In Revue française de sociologie, XXXII-I.

MORIN, E. (1977). La méthode, Tome I. La nature de la nature, Paris : Le Seuil.

PAI, Y., ADLER, J.A. (1997). Cultural foundations of education, $2^{\text {nd }}$ Ed., New Jersey: Prentice Hall.

PASSIS-PASTERNAK, G. (1991). Faut-il brûler Descartes? Du chaos à l'intelligence artificielle: quand les scientifiques s'interrogent. coll. Sciences et société, Paris : La Découverte.

PERRENOUD, P. (1996). Enseigner : agir dans l'urgence, décider dans l'incertitude, Paris : ESF Éditeur.

PRIGOGINE, I (1990). Visions : le chaos par Prigogine, In Actuel, nº 136, Paris. 